\title{
Combined system of precise positioning of pilotless helicopter-type aircraft in the landing area
}

\author{
Andrey Antonov ${ }^{1}$, Andrey Zubyuk ${ }^{2}$, Mikhail Kuznetsov ${ }^{1}$, Ilya Obukhov ${ }^{1}$, \\ Elizaveta Smirnova ${ }^{2, *}$, Egor Fadeev ${ }^{2}$, Leonid Chelyschev ${ }^{3}$, and Oleg Chikalo ${ }^{3}$ \\ ${ }^{1}$ SPA Sinergetika, 115419 Moscow, Russian Federation \\ ${ }^{2}$ M.V. Lomonosov MS, Physics Department, 119991 Moscow, Russian Federation \\ ${ }^{3}$ JSC NPP Radiotekchnika, 127083, Moscow, Russian Federation
}

\begin{abstract}
On-board equipment, ground equipment, and special software have been developed and tested that makes it possible to determine with high accuracy the relative coordinates of a helicopter-type unmanned aerial vehicle relative to a quick deployment runway in the absence of signals from global navigation systems.
\end{abstract}

\section{Introduction}

Unmanned aerial vehicles (UAVs) are not always in the area of reliable reception of signals from global navigation satellite systems (GNSS). In this regard, there is a need for accurate determination of UAV coordinates relative to the runway using autonomous means $[1,2]$.

UAVs can be used in hard-to-reach areas and dangerous for people conditions. The role of the runway can be played by any territory that is minimally suitable for taking-off and landing, not equipped with stationary navigation equipment. The system described here is equipped with mobile ground-based rapid deployment tools.

The proposed solution is intended for accurate positioning of a helicopter-type UAV (HT-UAV). Limitations are associated with the methods of landing and speed of vehicles $[3,4]$.

\section{System composition and purpose of components}

The system consists of radio and optical positioning subsystems, which complement or replace each other if necessary [4]. The composition of the system is shown in Fig. 1.

The radio subsystem allows the HT-UAV to detect the runway at a distance of $20 \mathrm{~km}$ and determine the relative bearing. At runway distances of less than $2 \mathrm{~km}$, it determines the horizontal distance to the landing point and the height above the landing point, the relative orientation of the HT-UAV and runway, and the true height of the HT-UAV.

* Corresponding author: smirnova.elizaveta@physics.msu.ru 
The optical subsystem can detect the runway at a distance of $1 \mathrm{~km}$ and determine the relative bearing. When approaching the runway at a distance closer than $100 \mathrm{~m}$ horizontally, this subsystem determines the height of the HT-UAV, the angular orientation of the HT-UAV concerning the runway and the relative coordinates of the HT-UAV and runway.

Figures 2 and 3 show the composition of the onboard and ground equipment. The sets and purpose of the modules of the radio engineering and optical subsystems are identical.

The module of reception of radio signals is realized on the basis of a cell of the receiving phased array antenna. The optical information reception module includes 16 IR photodiodes and three video cameras.

The input signal preprocessing module, the computational module, and the information exchange module with the on-board control system (BCS) provide processing of the input radio and optical information, calculate the relative coordinates of the HT-UAV, transmit data to the BCS and receive the necessary commands from the BCS.

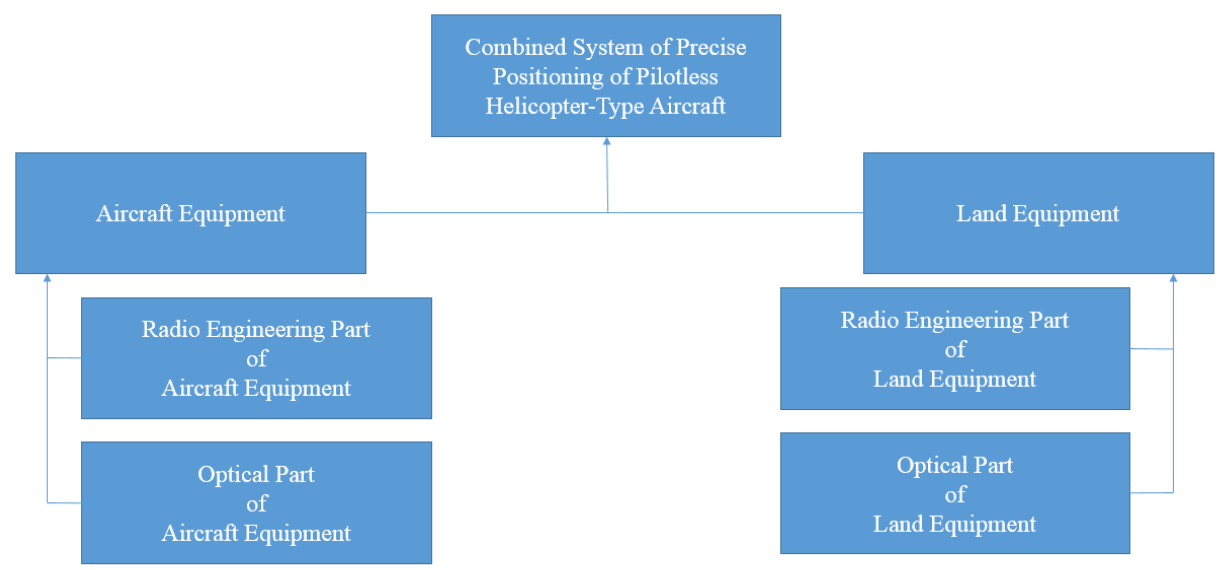

Fig. 1. Structure of the System.

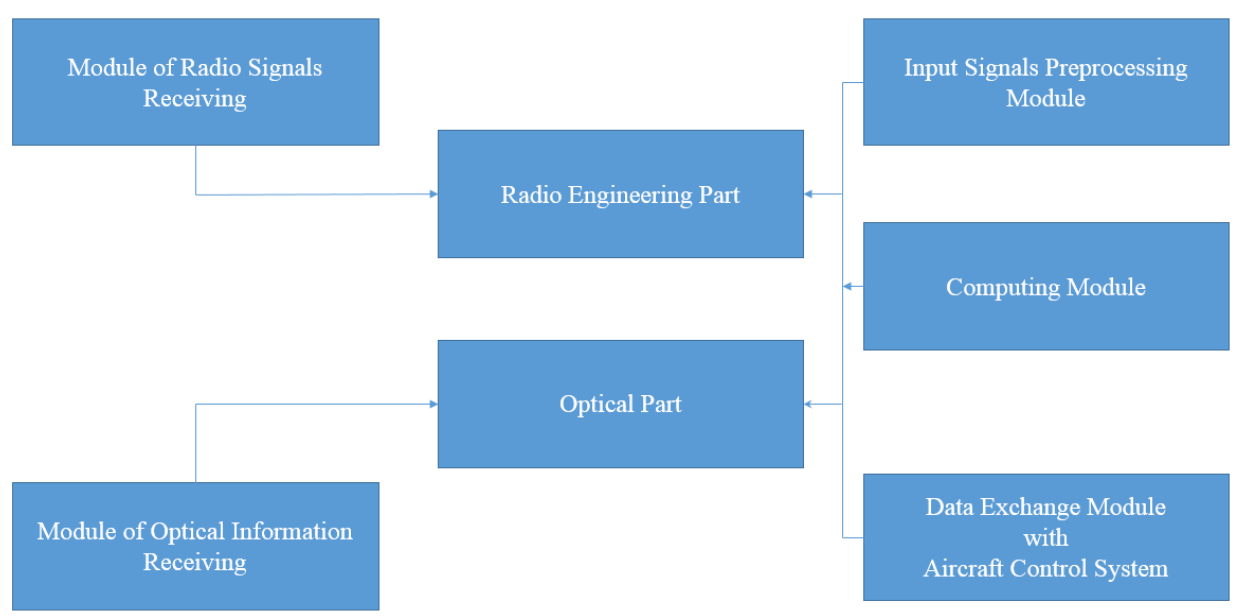

Fig. 2. Structure of the Onboard Equipment. 


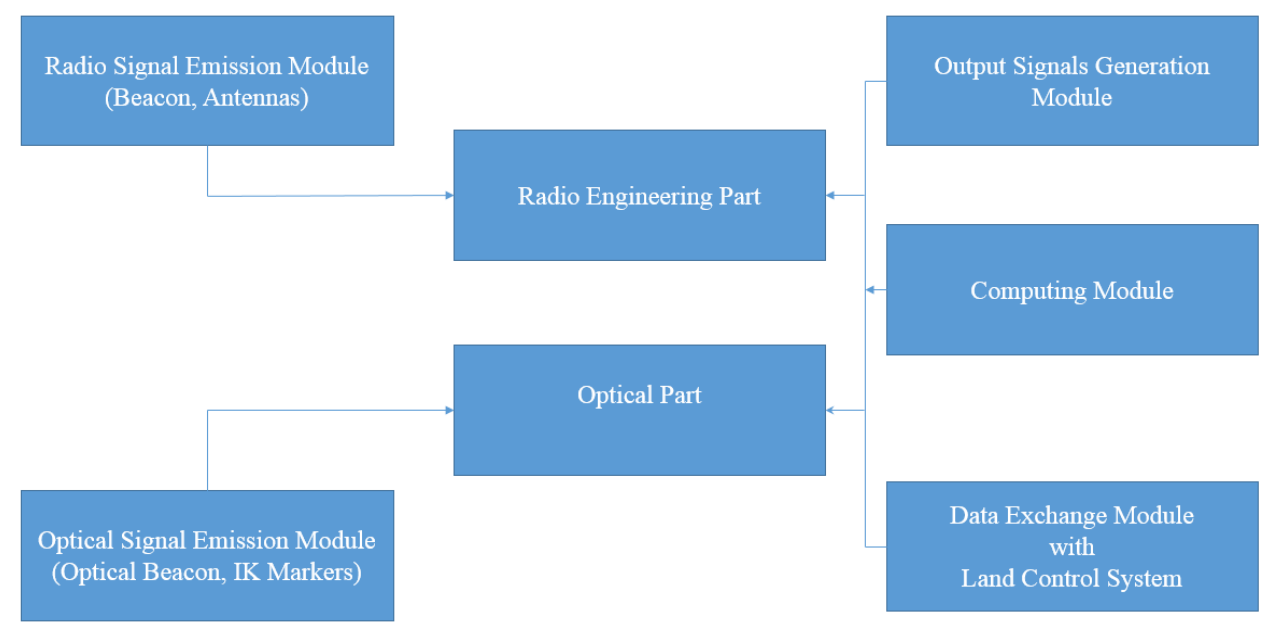

Fig. 3. Structure of the Ground Equipment.

Ground equipment includes a beacon; optical infrared beacon; optical and radio markers placed on the runway; output signal generation module; a computing module and an information exchange module with a ground control point (GCP).

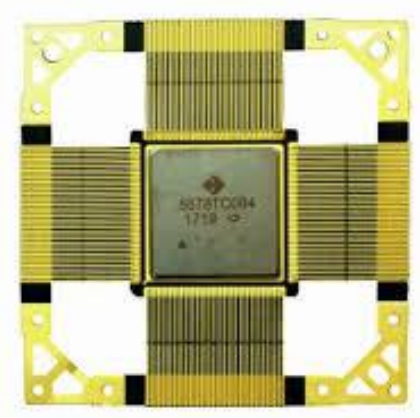

PLD 5578TC084 made by VZPP-S Company

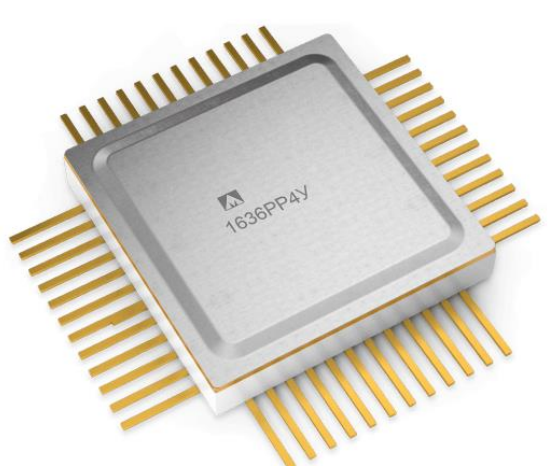

ROM made by MILANDR Company

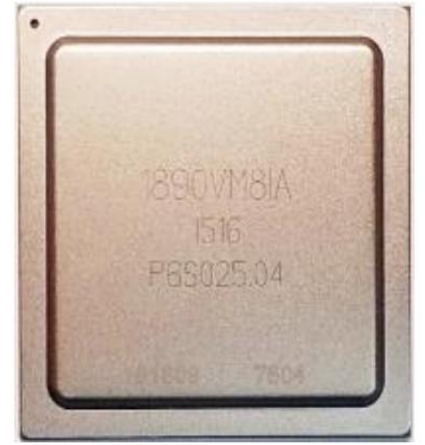

Processor 1890BM8Я designed by NIISI RAS

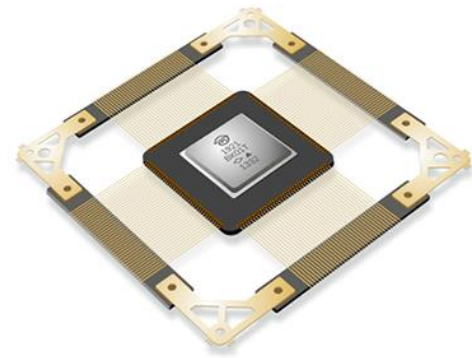

1921BK01T1

Microcontroller made by NIIET

Fig. 4. Some radio components produced in Russia and used in the System. 


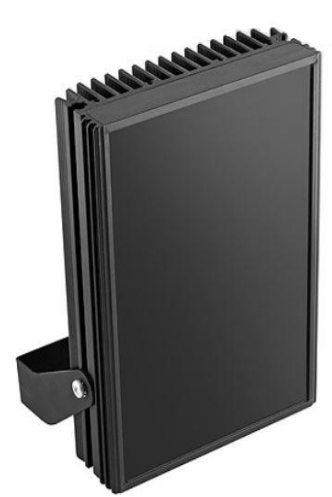

IR marker made by IR Technologies Manufacturing Company

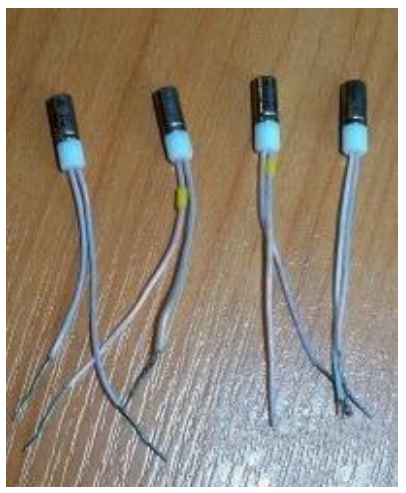

IR Photodiodes made by Moscow Plant "Sapfir"

Fig. 5. Some optical components produced in Russia and used in the System.

The system is equipped with completely original special software, including special software of controllers. Open-source software operates under the control of the Linux family operating system. PostgreSQL is used as a database management system.

The hardware of the system can be fully implemented on the basis of components manufactured in the Russian Federation, some of which are shown in Fig. 4 and 5.

\section{System testing}

The experimental sample and components of the system passed laboratory, field and flight tests, which confirmed the expected performance of the system.

The accuracy of the coordinates measurement by the radio subsystem was $0.05 \mathrm{~m}$, the accuracy of the speed measurement was $0.1 \mathrm{~m} / \mathrm{s}$.

The optical subsystem demonstrated the ability to determine the height with an accuracy of no worse than $5 \%$, the angular orientation of the HT-UAV with respect to the runway with an accuracy of no worse than $4^{\circ}$ and the relative coordinates of the HT-UAV and the runway with an accuracy of no worse than $2 \%$ of the true height value.

The original algorithms of technical vision used in the system have proven themselves well. They allow at distances up to $150 \mathrm{~m}$ to measure all the parameters required for HTUAV landing based on video streams from two spatially separated cameras installed on board, without using data from other system components.

The system provides the measurement of the required parameters by various methods and the calculation of their most reliable values for transmission to the GCP.

\section{Conclusion}

The developed Autonomous combined system of precise positioning of the HT-UAV on the basis of radio and optical components provides GCP with all the necessary information to drive the device to the landing zone from a distance of $20 \mathrm{~km}$ and landing on the rapid deployment runway.

The weight of the on-board equipment does not exceed $2.5 \mathrm{~kg}$. Its power is supplied from the on-board network of $27 \mathrm{~V}$. The weight of ground equipment does not exceed 25 $\mathrm{kg}$, it can be transported manually.

The system remains operational in the temperature range from $40^{\circ} \mathrm{C}$ to $+50^{\circ} \mathrm{C}$. 


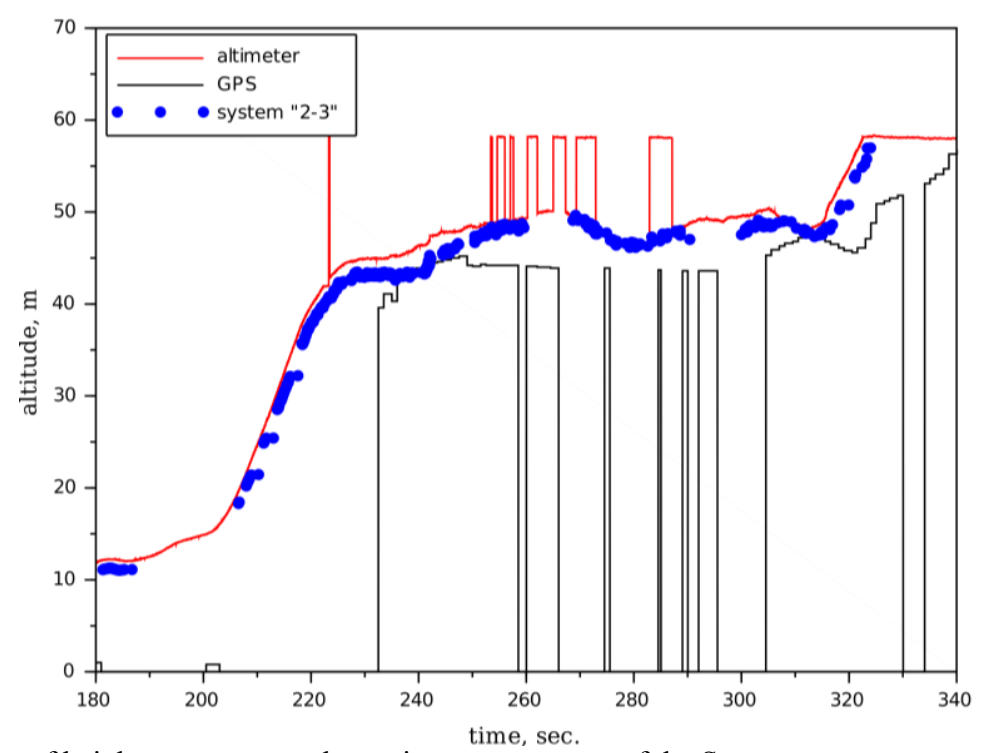

Fig. 6. Results of height measurement by various components of the System.

\section{References}

1. F. Alarcon, D. Santamaria, A. Viguria, UAV helicopter relative state estimation for autonomous landing on moving platforms in a GPS-denied scenario, IFACPapersOnLine, 48-9, pp. 37-42 (2015)

2. G.A. Krevetkin, Development of aircraft landing automation technology, Questions of defense engineering, 1 (253), pp. 36-42 (2012)

3. V.V. Scherbinin, G.A. Krevetkin, A.V. Sviyazov, V.B. Andrienko, Navigation support of unmanned aerial vehicles landing system, Gyroscopy and navigation, v. 1 (80), pp. 19-33 (2013)

4. V.A. Tupikov, V.A. Pavlova, G.E. Kaplinski, S.N. Krukov, Algorithm of automatic landing of unmanned aerial vehicle of helicopter type using onboard optoelectronic system, Proceedings of Tula state University. Technical science, v. 11 (3), pp. 86-95 (2016) 